NBER Working Paper Series

ASPECTS OF OPTIMAL UNEMPLOYMENT INSURANCE:

SEARCH, LEISURE AND CAPITAL MARKET IMPERFECTIONS

J. S. Flemming

Nuffield College, Oxford

Working Paper No. 214

CENTER FOR ECONOMIC ANALYSIS OF HUTAN BEHAVIOR

AND SOCIAL INSTITUTIONS

National Bureau of Economic Researcin, Inc. 204 Junipero Serra Boulevard, Stanford, CA 94305

November 1977

Preliminary; not for quotation.

NBER working papers are distributed informally and in limited number for coments only. They should not be quoted without written permission of the author.

This report has not undergone the review accorded official NBER publications; in particular, it has not yet been submitted for approval by the Board of Directors.

This research was supported by a contract w1th NBER from the Office of Income Security Policy, Department of Health, Education, and Welfare (No. HEW-100-76-0170). The author is grateful to Mart1n Bally, Margaret Bray, Martin Feldstein, Michael Hamer, Giuseppe Mazzarino, J1m Mirrlees and Aisthony Pellech1o, varlously for general discussion, for help with the stochastic processes, and for computing. 


\section{ASPECTS OF OPTIMAL UNEMPLOYMENT INSURANCE: SEARCH, LEISURE AND CAPITAL MARKET IMPERFECTIONS}

I

Unemployment Insurance (UI) can be seen as redistributive with a trade-off between equality and efficient 'search' to be set beside the existing 'effort' and 'education' models of optimal taxation (e.g., Mirrlees (1971), Atkinson (1973)). The redistributive role of UI is accentuated by capital market imperfections which tend to make consumption vary more closely with current income.

In this paper UI will be assumed to take the form of a proportional pay-roll tax used to pay a subsidy to all the unemployed.' However the analysis here, and elsewhere, raises a number of questions about this form of UI. The loss of a given amount of income will involve a larger utility loss (given a perfect capital market) for an older man who has fewer years over which to make the adjustment. Should he be given higher benefits? Shavell and Weiss (1977) argue that if benefits decline over time their disincentive effect is reduced; however Flemming (1977, b) has shown that if there is a progressive income tax on annual income, there is an efficiency argument for benefits increasing with duration. The incentive effects of UI depend on the mix of benefits in the traditional form of periodic payments conditional on continued unemployment, redundancy payments (either lump sums or unconditional payments for a set number of periods) (Hamermesh (1977)) and loans (the natural response to an imperfect capital market). Finally, as Baily (1977) mentions, subsidising search activities by the unemployed is another element in the potential policy mix.

Baily (1977) presents a two period model of the redistributive (insurance) argument for UI which involves a curious mixture of perfect and imperfect capital market assumptions. On the one hand, the 
unemployed can apparently not borrow at all against future earnings (i.e. earnings beyond the second period); on the other hand, the second period is not homogeneous and borrowing from the second (employed) section of it to finance consumption during unemployment is not only possible but costless. The framework is unsuited to the analysis of unemployment at the beginning of a career and is also inadequate for the identification of that part of a UI scheme justified by capital market imperfection.

The object of this paper is to examine the importance of capital market assumptions. A special continuous-time model is developed in sections II - IV which is applicable to the perfect capital market case. It can also be used when there is no capital market at all (section IV). For 'reasonable' parameter values the optimal replacement rate (ratio of benefits to gross wage) appears to be less than $20 \%$ when capital markets are perfect but over $70 \%$ when they are non existent (i.e. no saving or dis-saving).

These models follow Baily in ignoring leisure; however, in the perfect capital market case this produces some rather surprising results, e.g. the unemployed spend almost as much money on searching as they would earn if employed (and sometimes more). This result might be more plausible if the opportunity cost of an unemployed man's time were included in the costs of search, i.e. if leisure is valued. Sections VI and VII present results for a similar model with leisure (which has to enter in a rather special way). The result is that in the perfect capital market case the optimal replacement ratio falls to less than $5 \%$, while when there is no capital market it falls from $75 \%$ when leisure has zero value, to $45 \%$ when it is valued at one quarter of the wage, $25 \%$ at half the wage and $10 \%$ at three quarters of the wage. 
Just as portfolio theory with its multiplicative random rates of return is facilitated by the assumption of an iso-elastic or constant relative risk aversion utility function, so the analysis of a ranion: additive labour income stream is simplest if there is constant abeclute risk aversion. We therefore assume

$$
U(C)=-\alpha e^{-\beta C} \quad \alpha, \beta>0
$$

As an example of the stochastic dynamic programming techniques to be used subsequently we examine the following problem: given

(i) initial wealth $\mathrm{W}$;

(ii) the utility function (1);

(iii) a constant interest rate $r$;

(iv) labour income y generated by a stationary wiener process so that (undiscounted) income over any interval $h$ has mean and variance proportional to $h$;

(v) additive separability over an infinite horizon with pure time preference at the rate $\rho$.

What is the optimal consumption strategy?

Let $\phi(W)$ be the indirect utility function of wealth. Then

$$
\left.\begin{array}{c}
\phi(W)=\underset{C(W)}{\operatorname{Max}} \operatorname{Lim}_{h \rightarrow 0}\left\{h U(C(W))+(1-\rho h) E \phi\left(\tilde{W}_{+h}\right)\right\} \\
\tilde{W}_{+h}=(1+r h) W+\tilde{y}(h)-h C \\
\tilde{y}(h)=N(m h, v h)
\end{array}\right\}
$$

Expanding $\phi\left(W_{+h}\right)$ about $W$ and taking expectations (2) becomes 


$$
\phi(W)=\operatorname{Lim}_{h \rightarrow 0}\left(h U(C(W))+(1-\rho h)\left(\phi(W)+\phi^{\prime}(W)(r W+m-C) h\right.\right.
$$$$
+\phi^{\prime \prime}(W) v h / 2 \ldots+O\left(h^{2}\right)
$$

whence

$$
\rho \phi(W)=U(C(W))+\phi^{\prime}(W)(r W+m-C(W))+\phi^{\prime \prime}(W) v / 2
$$

Differentiating (3) w.r.t. W and C gives

$$
\left.\begin{array}{c}
\rho \phi^{\prime}=U^{\prime} \cdot C^{\prime}+\phi^{\prime}\left(r-C^{\prime}\right)+\phi^{\prime \prime}\left(r W^{\prime}+m-C\right)+\phi^{\prime \prime} \cdot v / 2 \\
U^{\prime}=\phi^{\prime}
\end{array}\right\}
$$

which reduce to

$$
C=r W+m+\frac{\phi^{\prime \prime \prime} v}{2 \phi^{\prime \prime}}-(\rho-r) \frac{\phi^{\prime}}{\phi^{n}}
$$

From (1) and (4)

$$
\begin{gathered}
\phi^{\prime}(W)=\alpha \beta e^{-\beta C(W)} \\
\phi^{\prime \prime}(W)=-\alpha \beta^{2} e^{-\beta C(W)} C^{\prime}(W) \\
\phi^{\prime \prime \prime}(W)=\alpha \beta^{3} e^{-\beta C(W)} C^{\prime}(W)^{2}-\alpha \beta^{2} e^{-\beta C(W)} C^{\prime \prime}(W)
\end{gathered}
$$

whence

$$
\frac{\phi^{\prime \prime \prime}}{\phi^{\prime \prime}}=-\beta C^{\prime}(W)+\frac{C^{\prime \prime}(W)}{C^{\prime}(W)} \text { while } \frac{\phi^{\prime}}{\phi^{\prime \prime}}=-\frac{1}{B C^{\prime}(W)}
$$

If $\phi^{\prime} / \phi^{\prime \prime}$ and $\phi^{\prime \prime} / \phi^{\prime \prime}$ were constants then $C^{\prime}=r$ and $C^{\prime \prime}=0$, in which case $\phi^{\prime \prime \prime} / \phi^{\prime \prime}=-B r$ and $\phi^{\prime} / \phi^{\prime \prime}=-\frac{1}{B r}$ which are indeed constants. Thus

$$
c=r W+m-\beta r v / 2+(\rho-r) / B r .
$$


is a solution to our problem (although we have not proved its uniqueness).

Equation (8) implies that negative financial wealth, which has not been ruled out, might involve negative consumption. This reflects the fact that the utility function (1) does not have the property that $U^{\prime} \rightarrow+\infty$ as $C \rightarrow 0 \quad\left(U^{\prime}(0)=\alpha \beta ; \quad U^{\prime}\right.$ goes to plus infinity only as $C$ goes to minus infinity). To impose a non-negativity constraint on $\mathrm{C}$ without changing $U(C), C>0$, would be both arbitrary and mathematically difficult but the present tractable mojel is certainly deficient in this respect.

Despite this deficiency equation (8) does enable us to identify a number of distinct potential effects of UI on welfare through its effects on the distribution or level of consumption.

(a) For a given distribution of wealti UI, if it raises unemployment, tends to lower mean labour income (m) but the effect of this on consumption may be offset by a reduction in the variance (v) of net income.

(b) In the longer run the distribution of wealth itself depends on $m$ and $v$; a reduction in the latter will reduce the inequality of wealth and consumption. Moreover by reducing savings a smaller $v$ reduces mean wealth and mean consumption unless offset by macro-policy.

(c) In a general equilibrium context, and in the absence of offsetting macro-policy, UI induced changes in accumulation and labour supply will lead to changes in interest rates and wages similar to the effects of other aspects of social security (see Fleming (1977)). If the budget is used to insulate capital accumulation from these effects UI wich raises unemployment must also lower mean consumption. 
(d) As $r \rightarrow 0$ the dependence of consumption on wealth and on the variance of labour income both decline. This suggests, as we shall see in subsequent calculations, that the level of optimal UI will be an incressing function of the real inteest rate tending to zero with the interest rate. However, UI does not, in the absence of offsetting macro-policy, increase monotonically with r. The effect of UI on savings, and thus wealth, has an effect on consumption proportionate to $r$.

\section{III}

The model of equations (1)-(8) cannot be used as it stands since it cannot easily admit of search activity undertaken by the unemployed. In the absence of search it is hard to obtain a disincentive effect of UI (indeed if one simply postulates that unemployment rises with UI replacement rates the optimum may be a tax on unemployment). Both search and compensation for the unemployed require that there be an identifiable unemployment state.

Consider therefore the following model, in which we follow Baily in ignoring labour/leisure choice; at any one time part of the population is employed and earns a gross wage of 1, part is unemployed and earns nothing. UI changes these numbers to $(1-t)$ and $d$ (for 'dole'). Balance of the UI fund implies

$$
t(1-u)=d u
$$

where $u$ is the unemployment rate.

A proportion $\lambda$ of those employed lose their job per unit time while a proportion $\varepsilon$ of the unemployed find jobs. We assume here that $\lambda$ is given by technological change etc. and is independent of 
UI. $\varepsilon$ depends on search strategy which is influenced by UI. Note that in the absence of population growth the steady state value of $u$ is $\lambda /(\lambda+\varepsilon)$ so that (9) becomes

$$
t=\lambda d / \varepsilon \text {. }
$$

We initially retain the infinite horizon assumption of the previous section in which case there are two indirect utility functions of wealth, one for each employment state, and two consumption functions. Using subscripts $e$ and $u$ for the states the following equation, comparable to (3) above, can easily be derived

$$
\left.\begin{array}{l}
\rho \phi_{e}(W)=U\left(C_{e}(W)\right)+\phi_{e}^{\prime} \cdot\left(r W+(1-t)-C_{e}(W)\right)+\lambda\left(\phi_{u}(W)-\phi_{e}(W)\right) \\
\text { and } \\
\rho \phi_{u}(W)=U\left(C_{u}(W)\right)+\phi_{u}^{\prime} \cdot\left(r W+d-s-C_{u}(W)\right)+\varepsilon\left(\phi_{e}(W)-\phi_{u}(W)\right)
\end{array}\right\}
$$

where $s$ is the amount of resources expended in search. Differentiating (11) w.r.t. $W$ and $C$,

$$
U^{\prime}\left(C_{i}(W)\right)=\phi_{i}^{\prime}(W) \quad i=e, u
$$

and

$$
\begin{gathered}
\lambda\left(\phi_{u}^{\prime}-\phi_{e}^{\prime}\right)=-\phi_{e}^{\prime \prime}\left(r W+(1-t)-c_{e}(W)\right)+(\rho-r) \phi_{e}^{\prime} \\
\varepsilon\left(\phi_{u}^{\prime}-\phi_{e}^{\prime}\right)+(\rho-r) \phi_{u}^{\prime}=\phi_{u}^{\prime \prime}\left(r W+d-s-c_{u}(W)\right)
\end{gathered}
$$

Initially we would expect $C_{e}(W)>C_{u}(W)$ with less than perfect insurance and positive interest, thus we expect $\phi_{u}^{\prime}>\phi_{e}^{\prime}$ and the LHS of (12) positive while $\phi^{\prime \prime}$ is always negative. (12) therefore implies, in the case in which $\rho=r$, 


$$
\left.\begin{array}{l}
c_{e}(W)<r W+(1-t) \\
c_{u}(W)+s>r W+d
\end{array}\right\}
$$

As one would expect, the employed save while the unemployed dissave.

Differentiating (11) w.r.t. s gives

$$
\varepsilon_{s}=\phi_{u}^{\prime} /\left(\phi_{e}-\phi_{u}\right)
$$

Arguments similar to those used above show that a solution to the optimal consumption problem takes the form

$$
c_{e}=r w+y_{e}-\gamma_{e}, \quad c_{u}=r W+y_{u}-\gamma_{u}
$$

where

$$
y_{e}=1-t, \quad y_{u}=d-s
$$

and

$$
\begin{aligned}
& \gamma_{e}, \gamma_{u} \text { are constants (which will be referred) } \\
& \text { to as 'savings') }
\end{aligned}
$$

It follows that, for the utility function (1), dropping the subscript,

$$
\left.\begin{array}{c}
\phi^{\prime}=\alpha \beta e^{-\beta C} \\
\phi^{\prime \prime}=-\alpha \operatorname{\beta r} e^{-\beta C}
\end{array}\right\}
$$

whence, assuming for convenience that $\rho=r$, (12) can be written as

$$
\left.\begin{array}{l}
\lambda\left(e^{\beta\left(\left(y_{e}-\gamma_{e}\right)-\left(y_{u}-\gamma_{u}\right)\right)}-1\right)=\beta r \gamma_{e} \\
\varepsilon\left(1-e^{-\beta\left(\left(y_{e}-\gamma_{e}\right)-\left(y_{u}-\gamma_{u}\right)\right)}\right)=-\beta r \gamma_{u}
\end{array}\right\}
$$


whence

$$
\varepsilon \gamma_{e}+\lambda \gamma_{u}=-\beta r \gamma_{e} \gamma_{u}
$$

i.e.

$$
\begin{gathered}
\gamma_{e}=-\lambda \gamma_{u} /\left(\varepsilon+\beta r \gamma_{u}\right) \\
\gamma_{u}=-\varepsilon \gamma_{e} /\left(\lambda+\beta r \gamma_{e}\right) \\
\gamma_{e}-\gamma_{u}=-\gamma_{u}\left(1+\frac{\lambda}{\varepsilon+\beta r \gamma_{u}}\right)=\gamma_{e}\left(1+\frac{\lambda}{\varepsilon+\beta r \gamma_{e}}\right)
\end{gathered}
$$

so that (17) becomes, writing $\delta$ for $y_{e}-y_{u}$,

$$
\begin{aligned}
& \left.\begin{array}{rl}
\delta & =(1-t)-(d-s) \\
& =1+s-d(\lambda+\varepsilon) /(\varepsilon)
\end{array}\right\} \\
& \lambda\left(e^{\beta\left(\delta-\gamma_{e} e^{\left(\frac{\lambda+\varepsilon+\beta r \gamma_{e}}{\lambda+\beta r \gamma_{u}}\right)}\right)}-1\right)=\beta r \gamma_{e}
\end{aligned}
$$

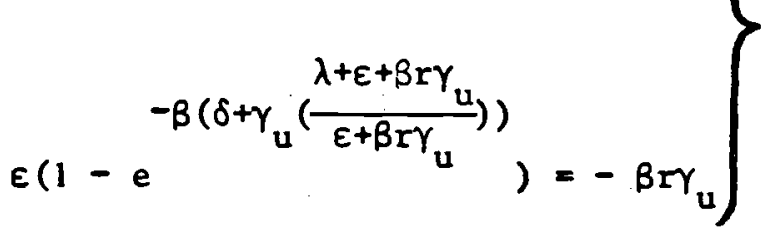

or

$$
\left.\begin{array}{l}
\beta\left(\delta-\gamma_{e}\left(\lambda+\varepsilon+B r \gamma_{e}\right) /\left(\lambda+\beta r \gamma_{e}\right)\right)=\log \left(\left(\varepsilon+\beta \Gamma \gamma_{e}\right) / \lambda\right) \\
\beta\left(\delta+\gamma_{u}\left(\lambda+\varepsilon+\beta r \gamma_{u}\right) /\left(\varepsilon+\beta r \gamma_{u}\right)\right)=-\log \left(\left(\varepsilon+\beta r \gamma_{u}\right) / \varepsilon\right)
\end{array}\right\}(21)
$$

$$
\left.\begin{array}{l}
\gamma_{e}=\frac{\lambda \delta}{\lambda+\varepsilon}=u \delta \\
-\frac{\varepsilon \delta}{\lambda+\varepsilon}=-(1-u) \delta
\end{array}\right\}
$$


Returning to (14) it can now be rewritten, using (17), as

$$
\varepsilon_{s}=r \beta\left[1-e^{-\beta\left(\delta-\left(\gamma_{e}-\gamma_{u}\right)\right)}\right]^{-1}=-\varepsilon / \gamma_{u}
$$

If we assume that there is no externality to search we might consider the following model of the determination of $\varepsilon$.

The $i^{\text {th }}$ unemployed man's chance of finding a job in a unit period is

$$
\varepsilon_{i}=n_{i} p
$$

where $n_{i}$ is the number of contacts per period and $p$, their success rate, is given by

$$
p=\frac{h}{n u}
$$

where $h$, the number of hirings per period, is equal to the number of job losses, i.e. ${ }^{2}$

$$
h=\lambda(1-u)
$$

Thus

$$
\varepsilon_{i}=\frac{n_{i}}{n} \lambda \cdot \frac{(1-u)}{u}=\varepsilon \frac{n_{i}}{n}
$$

$n_{i}$, the number of contacts per period, is a function of the intensity of search, for simplicity we assume

$$
n_{i}=88_{i}^{n}
$$

where $\eta<1$ indicates diminishing returns (rising travel costs?). The individual takes average $n\left(=g s^{n}\right)$ as given and thus chooses $s_{i}$ to maximize $\phi_{u}\left(W_{i}\right)$ subject to 


$$
\varepsilon_{i}=\varepsilon\left(\frac{s}{s}\right)^{n}
$$

whence, at the optimum

$$
\varepsilon_{i_{s_{i}}}=n \varepsilon\left(\frac{s_{i}}{s}\right)^{n} / s_{i}
$$

Since in equilibrium $s_{i}=s$

$$
\varepsilon_{s}=\eta \frac{\varepsilon}{s}
$$

and equating (23) and (31) gives

whence

$$
\left.\begin{array}{l}
s=-n r_{u} \\
n p=g p\left(-n r_{u}\right)^{n}
\end{array}\right\}
$$

where $p$, the success rate, is assumed to be exogenously fixed by the behaviour of employers. ${ }^{3}$

Equations (32) imply that search, and the duration of spells of unemployment, are both independent of wealth. This independence enormously simplifies the dynamics of wealth.

Some idea of the structure of the model can be obtained if we consider the case of $p=r=0$. As was suggested in relation to equation (8), and is confirmed below, the optimal dole is zero if $\mathbf{r}=0$. In this case,

from (19)

$$
\delta=1+8
$$

from (32)

$$
s=-\eta \gamma_{u}
$$

from (22) 


$$
\begin{aligned}
\gamma_{u} & =-\varepsilon \delta /(\lambda+\varepsilon)=-\varepsilon(1+\varepsilon) /(\lambda+\varepsilon) \\
& =\frac{-\varepsilon}{\lambda+\varepsilon}\left(1-\eta \gamma_{u}\right) \\
& =\frac{-\varepsilon}{\lambda+\varepsilon(1-\eta)} \\
\varepsilon & =g p\left(\frac{\varepsilon \eta}{\lambda+\varepsilon(1-\eta)}\right)^{n} \\
\gamma_{e} & =\frac{\lambda}{\lambda+\varepsilon(1-\eta)}
\end{aligned}
$$

If $\varepsilon$ is much greater than $\lambda$

$$
\varepsilon \approx \operatorname{gp}\left(\frac{\eta}{1-\eta}\right)^{n}
$$

In the next section we take $n=0.5$ as the central case, in which case $\varepsilon \approx$ gp. We shall also take $\lambda=0.2$ as a central case (i.e. a mean duration of employment spells of five years). Thus if the unemployment rate $\frac{\lambda}{\lambda+\varepsilon}$ were $2 \%$ in the absence of UI it would imply $g P=9.8$. We therefore take $g \mathrm{p}=10$ as a central case so that $\varepsilon \approx 10$ (mean duration of a spell of unemployment 5.2 weeks). More precisely, assuming $r=\rho=d=0, \quad g p=10, \quad \eta=0.5, \lambda=0.2$ implies $\varepsilon=9.8$, $\gamma_{u}=-1.92, \gamma_{e}=0.04, s=0.96, u=0.02$. Thus the unemployed dissave 1.92 times their wage, spending half of this on search and consuming the other half; as mentioned in the introduction, this possibly impl ausible result is modified in the model of sections VI. and VII which includes leisure.

At 0.96 the consumption of the unemployed is identical to that of the employed. This reflects the fact that in the absence of discounting and with an infinite horizon the unemployed have the same human capital as the employed while financial assets/liabilities are irrelevant. It 
is the equality between the two groups which makes UI redundant in this case.

Social Welfare (S), our maximand, depends on the level and distribution of consumption. Suming the individual instantaneous utilities (1) gives

$$
\begin{aligned}
S= & -\alpha \int \exp (-\beta \cdot C(W)) \cdot f(W) d W \\
= & \alpha\left[(1-u) \int \exp \left(-\beta, C_{e}(W)\right) \cdot f_{e}(W) d W-u \int \exp \left(-\beta(a(W)) \cdot f_{u}(W) d W\right]\right. \\
& \text { If the distributions } f_{e}(W), f_{u}(W) \text { were both normal, } C_{e}(W) \text { and }
\end{aligned}
$$
$C_{u}(W)$ would also be normal so that individual utilities would be lognormally distributed and the aggregate welfare could be written as

$$
S=\frac{-\alpha}{\lambda+\varepsilon} \cdot\left\{\varepsilon \exp \left(-\beta \bar{C}_{e}+\beta^{2} r^{2} v_{e} / 2\right)+\lambda\left(-\beta \bar{C}_{u}+\beta^{2} r^{2} v_{u} / 2\right)\right\}
$$

where $v_{e}$ and $v_{u}$ are the variances of $f_{e}(W)$ and $f_{u}(W)$ while $\bar{c}_{e}$ and $\bar{c}_{u}$, the mean consumption levels of the employed and unemployed respectively are, from (15):

$$
\bar{C}_{i}=E_{i}(w)+Y_{i}-Y_{i} \quad i=e, u
$$

It is thus necessary to establish the distributions $f_{e}(W)$ and $f_{u}(W)$. In the limit, as time goes to infinity, the distributions have infinite means and variances since both are proportional to time. Indeed eventually the two distributions become indistinguishable with 


$$
V(W(T))=2 T\left(\gamma_{e}-\gamma_{u}\right)^{2} \lambda \varepsilon /(\lambda+\varepsilon)^{3}
$$

and

$$
E(W(T))=W(0)+T\left(\varepsilon \gamma_{e}+\lambda \gamma_{u}\right) /(\lambda+\varepsilon)
$$

of course individuals do not have infinite lives. If we consider finite lives the consumption optimization conditions change in three ways: where we have $\mathrm{rW}$ alone we will find $W \cdot a(r, \tau)$ - the arnuity value of $W$ at $r$ for the $\tau$ years to the horizon; the savings rates $\gamma_{e}$ and $\gamma_{u}$ though still independent of $W$ vary with $\tau$, as also does optimal search s - search, like saving, being less worthwhile the nearer the agent is to his horizon. These features of the finite horizon case make the dynamics of wealth very much more difficult to handle than in the infinite case.

For tractability, therefore, we are restricted to the latter for which we evaluate below the instantaneous social welfare of a population of immortals at an arbitrary date $T \cdot$ after they set out on the process with equal weal th $W(0)$ and the equilibrium distribution of unemployment (which depends on the UI regime). If $T$ is large enough we can substitute from (36) into (35) to get

$$
\begin{aligned}
& S(T) \approx \frac{-\alpha}{\lambda+\varepsilon}\left\{\exp \left[\frac{\beta r T}{\lambda+\varepsilon} \beta r \frac{\left(\gamma_{e}-\gamma_{u}\right)^{2} \lambda \varepsilon}{(\lambda+\varepsilon)^{2}}-\left(\varepsilon \gamma_{e}+\varepsilon \gamma_{u}\right)\right] .\right. \\
& {\left[\varepsilon \operatorname { e x p } \left(-\beta\left(1-\lambda d / \varepsilon-\gamma_{e}\right)+\lambda \exp \left(-\beta\left(d-(1-\eta) \gamma_{u}\right)\right]\right.\right.}
\end{aligned} .
$$

Equation (21) gives $\gamma_{u}$ as a function of the parameters $B, \quad r$ and $\lambda$ and the endogenous variables $\delta$ and $\varepsilon$. From (19) $\delta$ depends on the parameters $\lambda$ and $d$ and the endogenous variables $s$ and $\varepsilon$. From (32) $s=-\eta \gamma_{u}$ (where $\eta$ is a parameter) and $\varepsilon=$ gps ${ }^{\eta}$ where $g$ 
and $p$ are parameters. Thus suitable substitution enables us to solve (21), (32) and (19) for $\gamma_{u}, \varepsilon, \delta$ (and s); (21) can then be solved for $\gamma_{e}$ and then, given $T$ and $\alpha$ we can evaluate (37) for $S(T)$. The optimal value of $d$ was found by searching over a $1 \%$ grid; in addition to the values of $\gamma_{i}, \varepsilon, s, u$, and $t$ the variance of wealth factor $V=\left(\gamma_{e}-\gamma_{u}\right)^{2} \lambda \dot{\varepsilon} /(\lambda+\varepsilon)^{3}$ was calculated and also the contribution ( $x$ ) of accumulated risk induced savings (remember $r=\rho$ throughout $), \quad x \equiv \operatorname{Tr}\left(\varepsilon \gamma_{e}+\lambda \gamma_{u}\right) /(\lambda+\varepsilon)$ and also the ratio of the consumption of the employed to that of the unemployed (at $W=0$ ) $z \equiv\left(d-\left(\gamma_{u}+s\right)\right) /\left(1-t-\gamma_{e}\right)$.

As described above we took $\lambda=0.2, \quad n=0.5$ and $g p=10$ as the standard values of those parameters. Since most people work for about 40 years we took $T=20$ for the standard case although with a mean duration of five years for employment spello it is rather short for our asymptotic approximation of the wealth distribution. The standard value of $r(=\rho)$ was taken as $10 \%$ per annum; as a net real rate this is on the high side but it should be biased upwards to make some allowance for finite lives. Given our wage unit consumption is near to unity so that $B$ measures both absolute and relative risk aversion; we take $B=2$ as standard.

Most parameters were also tried at half and twice the standard value; with three alternative values of each of six parameters we would have $729\left(=3^{6}\right)$ combinations. This number reduces to 13 if only one parameter at a time is allowed to deviate from its standard value.

In the standard case average saing in response to earnings uncertainty is, in the absence of UI, $0.15 \%$ of the gross wage, accumulated for 20 years this adds $3.0 \%$ of the wage to mean wealth (remember that the accruing interest is all consumed) and thus at the $10 \%$ standard interest rate, adds $0.3 \%$ of the wage to mean consumption. In this case the error 
introduced by our partial analysis is trivial. However when $r=50 \%$ this factor adds a non-trivial $8 \%$ of the wage to mean consumption in the absence of UI which falls to $5 \%$ when $d=30 \%$. For this reason $x$ is included in the table below, it represents the addition to mean consumption jepardized by UI. $x\left(d^{*}\right)$ is also reported; if $x(0)-x\left(d^{*}\right)>0.2 \%$ of the wage the savings effect is likely to have a significant impact on the estimate of the optimal level of UI. In these cases we also evaluate the optimal UI when its effect on private savings is suppressed.

The results of these calculations are set out in table 1 below. Section (i) of the table sets out the different combinations of parameters in rows $1-13$ (rows 14 and 15 repeat rows 4 and 5 but the optimization (in section (iii)) is with the savings effect suppressed). Section (ii) gives the values of $\varepsilon, s, u, z, x, v$ etc. in the absence of UI $\varepsilon$ while section (iii) gives the optimal values of $d$ etc. for these parameters while the impact of optimal UI on search, unemployment and the variance of wealth is set out in section (iv).

Row 3 of the table is the standard case. In the absence of UI is 9.7 so that $u=\frac{\lambda}{\lambda+\varepsilon}=\frac{.2}{9.9}=2 \% . \varepsilon=9.7$ implies a mean duration of spells of unemployment of 5.4 weeks while the unemployed spend $94 \%$ of the gross wage on search and consume the same amount. The high search expenditures are more sensitive to $\eta$ than to gp (see rows $10,11,13)$. In the standard case the optimal 'dole' is $9 \%$ of the gross wage, this raises unemployment by $5 \%$ to $2.1 \%$ and can be financed by a $0.2 \%$ tax on the employed. The 'dole' raises the consumption of the unemployed from $94 \%$ to $95 \%$ of the wage and reduces the variance of wealth by $7 \%$. This reflects the fall of $\left(\gamma_{e}-\gamma_{u}\right)$ from $192 \%$ to $178 \%$ of the wage, half of the reduction coming from a cut in search expenditures and half from the direct effect of the dole on dissaving. 


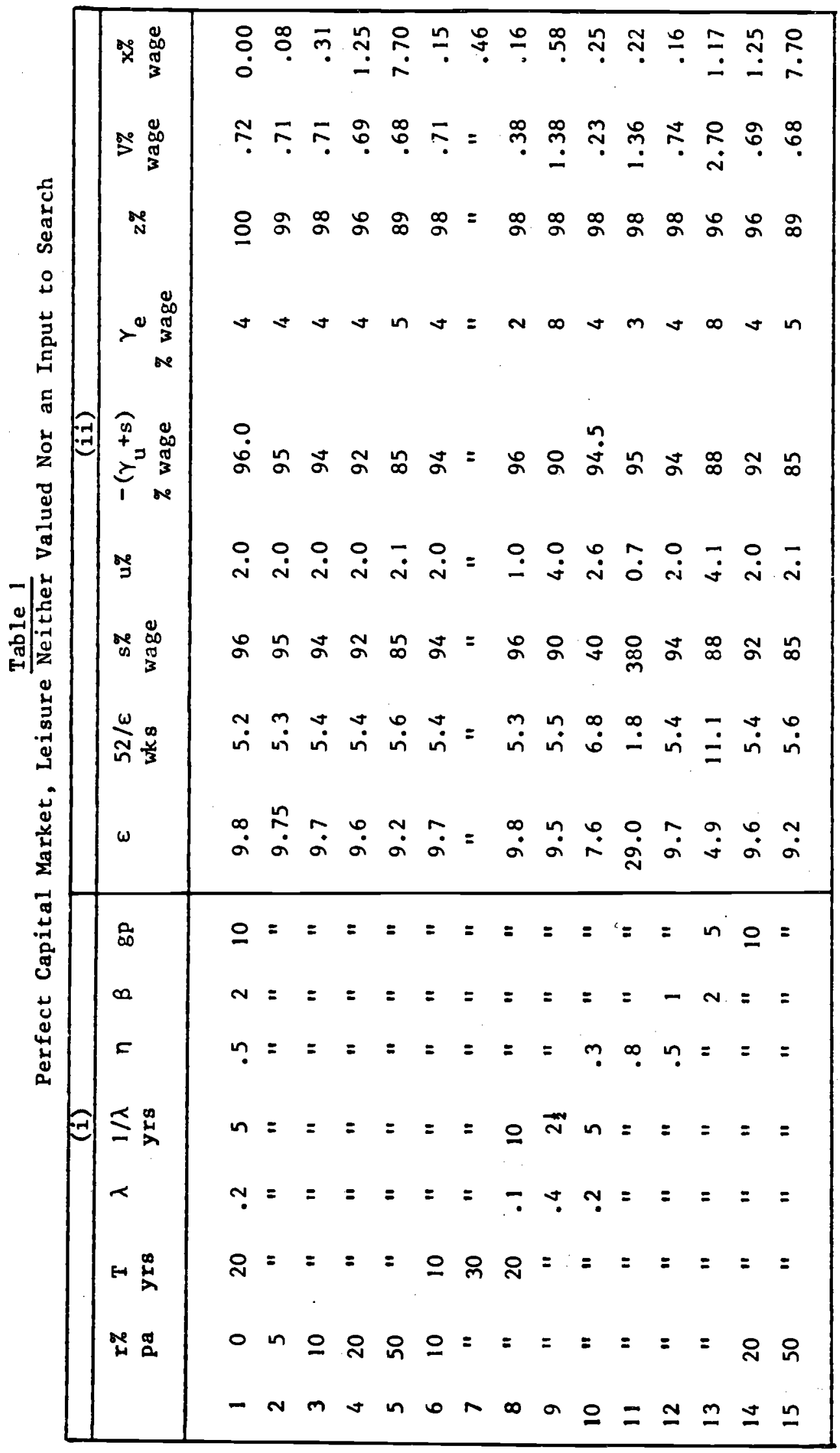




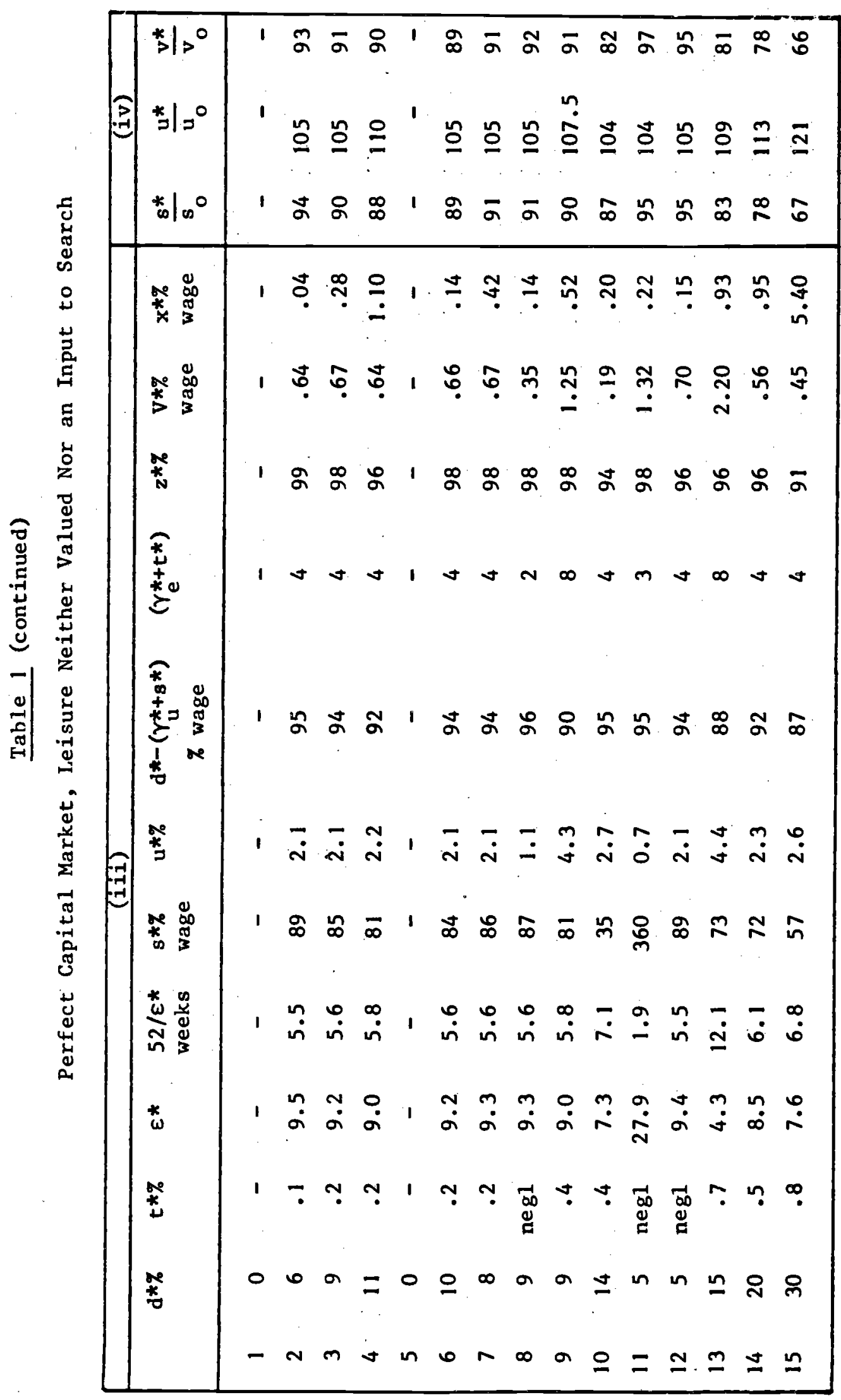


Conparison of rows 14 and 15 with 4 and 5 reemphasizes the importance of the savings effect when $r$ is high. Suppressing this effect doubles the optimal 'dole' at $r=20 \%$. Rows 3, 8 and 9 show that changing $\lambda$ changes only the unemployment rates (proportionately).

In the absence of a capital market things are much simpler:

$$
\begin{aligned}
& \left.\begin{array}{l}
c_{e}=1-t \\
c_{u}=d-s \\
t=\lambda d / \varepsilon
\end{array}\right\} \\
& \rho \phi_{e}=U\left(C_{e}\right)+\lambda\left(\phi_{u}-\phi_{e}\right) \\
& \left.\begin{array}{c}
\rho \phi_{u}=U\left(C_{u}\right)+\varepsilon\left(\phi_{e}-\phi_{u}\right) \\
U_{u}^{\prime}=\varepsilon^{\prime}\left(\phi_{e}-\phi_{u}\right)
\end{array}\right\} \\
& \text { If } U(.)=-\alpha e^{-B C} \text { as above, then } \\
& \left.\phi_{e}=\left(\lambda \phi_{u}-\alpha e^{-\beta(1-t)}\right)(\rho+\lambda)^{-1}\right)
\end{aligned}
$$

while

and (by (31))

$$
\left.\begin{array}{c}
\varepsilon^{\prime}=\frac{\alpha \beta e^{-\beta(d-s)}}{\phi_{e}}=\phi_{u} \\
\varepsilon^{\prime}=n \varepsilon / s
\end{array}\right\}
$$


From (40)

$$
\left.\begin{array}{l}
\phi_{e}=-\frac{\alpha}{\rho} \frac{(\rho+\varepsilon) e^{-\beta(1-t)}+e^{-\beta(d-s)}}{\rho+\lambda+\varepsilon} \\
\phi_{u}=-\frac{\alpha}{\rho} \frac{(\rho+\lambda) e^{-\beta(d-s)}+e^{-\beta(1-t)}}{\rho+\lambda+\varepsilon}
\end{array}\right\}
$$

and

$$
\phi_{e}-\phi_{u}=-\alpha\left(e^{-\beta(1-t)}-e^{-\beta(d-s)}\right)(\rho+\lambda+\varepsilon)^{-1}
$$

whence (from (41))

$$
\varepsilon^{\prime}=\beta(\rho+\lambda+\varepsilon)\left(1-\mathrm{e}^{-\beta \delta}\right)^{-1}=\eta \varepsilon / \mathrm{s}
$$

whence, as in (19).

$$
\delta=(1-t)-(d-s)=1+s-d(\lambda+\varepsilon) / \varepsilon
$$

Social welfare in this model is

$$
S=-\alpha\left(\lambda e^{-\beta(d-s)}+\varepsilon e^{-\beta(1-t)}\right) /(\lambda+\varepsilon)
$$

while

$$
\left.\begin{array}{c}
\beta \delta=-\log (1-\beta s(\rho+\lambda+\varepsilon) / n \varepsilon) \\
\varepsilon=\mathrm{gps}^{n}
\end{array}\right\}
$$

Table 2 sets out the results of maximizing (44) subject to these constraints. 


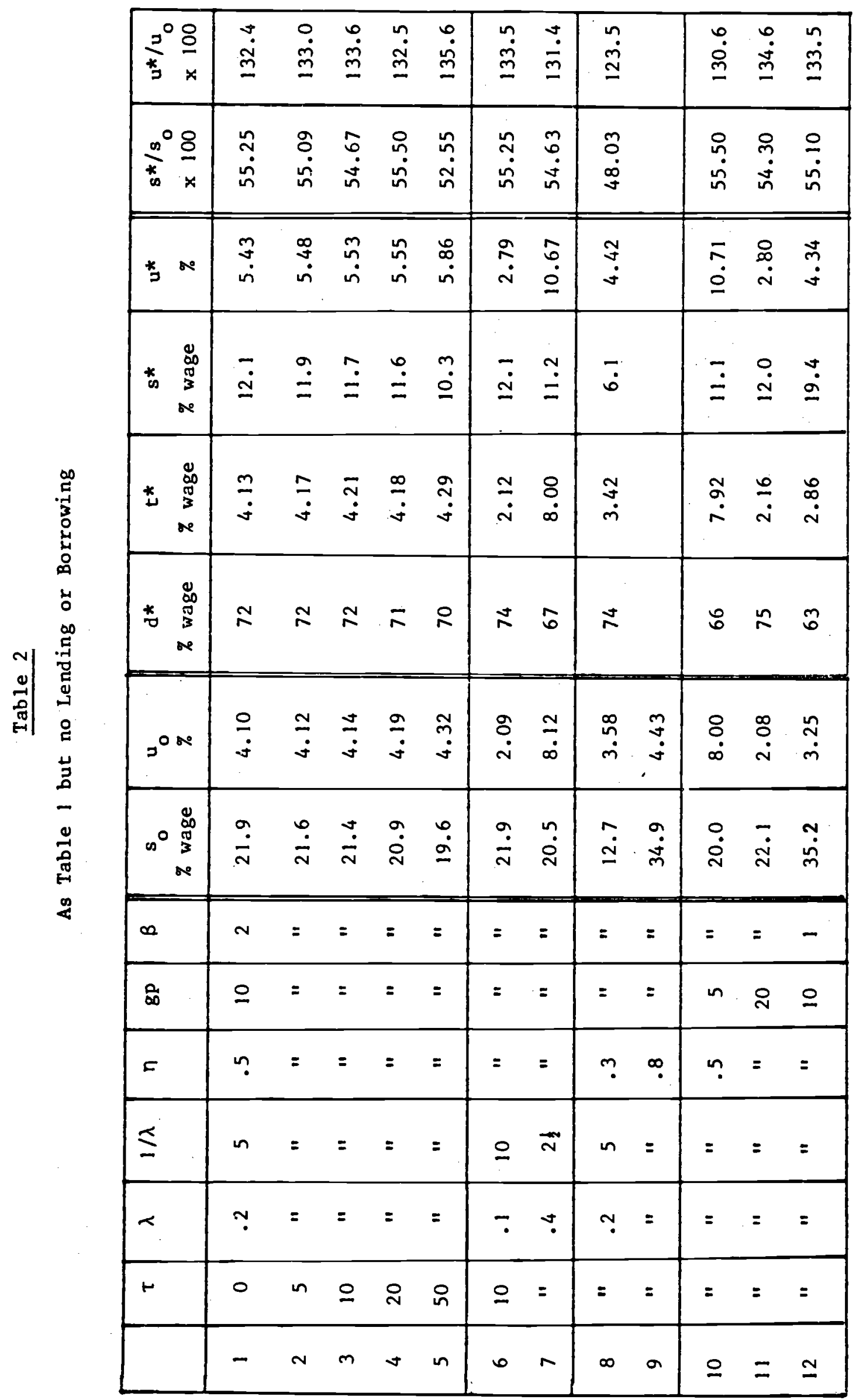


VI

In practice leisure rather than wealth is what is used up in search as mentioned in the introduction. It is important for the tractability of the model that the intensity of search be independent of wealth; since consumption depends on wealth this means that we need a utility function with constant relative risk aversion in consumption and the marginal rate of substitution between consumption and leisure independent of the level of consumption.

The function must be

$$
U(C, h)=-\alpha e^{-\beta(C+\theta(1-h))}
$$

where the consumer is endowed with one unit of useful time (per period) and $h$ is the proportion of it devoted to work (l) or search (s). With a wage rate of unity labour income is $l$ so that it follows from (46) that $\ell *=0,1$, as $\theta \gtrless 1$.

We assume $\theta<1$ so that given the opportunity people work fulltime. Search, unlike work, is subject to diminishing returns so that $\mathrm{s}^{*}$ will be interior. With a dole for the wholly unemployed, work/is only worthwhile if $1-t>\theta+d$ for which $d<1-\theta$ is necessary but not sufficient.

With this change equations (11) become

$$
\begin{aligned}
& \rho \phi_{e}(W)=U\left(C_{e}(W), 0\right)+\phi_{e}^{\prime}\left(r W+(1-t)-C_{e}(W)\right)+\lambda\left(\phi_{u}(W)-\phi_{e}(W)\right) \\
& \rho \phi_{u}(W)=U\left(C_{u}(W),(1-s)\right)+\phi_{u}^{\prime}\left(r W+d-C_{u}(W)\right)+\varepsilon\left(\phi_{e}(W)-\phi_{u}(W)\right)
\end{aligned}
$$

where by differentiation w.r.t. $C, W$ and $s$, and setting $\rho=r$ 


$$
\left.\begin{array}{c}
U_{i c}=\phi_{i}^{\prime} \quad i=e, u \\
\lambda\left(\phi_{u}^{\prime}-\phi_{e}^{\prime}\right)=-\phi_{e}^{\prime \prime}\left(r W+(1-t)-c_{e}(W)\right) \\
\varepsilon\left(\phi_{u}^{\prime}-\phi_{e}^{\prime}=\phi_{u}^{\prime \prime}\left(r W+d-c_{u}(W)\right)\right. \\
-\partial U\left(C_{u}(W),(1-s)\right) / \partial s=\varepsilon_{s}\left(\phi_{e}-\phi_{u}\right)
\end{array}\right\}
$$

which correspond to equations (11), (12) and (14).

We now define $y_{u} \equiv d$ and note that

$$
\begin{gathered}
\phi^{\prime}=\alpha \beta \mathrm{e}^{-\beta(C+\theta(1-h))} \\
\phi^{\prime \prime}=-\alpha r \beta^{2} \mathrm{e}^{-\beta(C+\theta(1-h))}
\end{gathered}
$$

and find that although the exponents in equation (17) now involve the term $-\theta(1-s)$ equations $(18),(20),(21)$ and (22) are unchanged except that $\delta$ (in equation (19)) is now given by

$$
\begin{aligned}
\delta & =1-t-d-\theta(1-s) \\
& =1-\theta+\theta s-d(\lambda+\varepsilon) / \varepsilon
\end{aligned}
$$

Using the utility function (46) (49) becomes

$$
\begin{aligned}
& \varepsilon_{s}=\alpha \beta \theta e^{-\beta\left(C_{u}+\theta(1-s)\right)} /\left(\phi_{e}-\phi_{u}\right) \\
& =-r \beta \theta e^{-\beta\left(C_{u}+\theta(1-s)\right)} /\left(e^{-\beta C} e-e^{\beta\left(C_{u}+\theta(1-8)\right)}\right) \\
& =r \beta \theta /\left(1-e^{-\beta\left(C e^{-C} u^{-\theta(1-s))}\right)}\right.
\end{aligned}
$$

whence, using the modified version of (17)

$$
\varepsilon_{\mathbf{s}}=-\varepsilon \theta / \gamma_{\mathbf{u}}
$$


Equations (24)-(31) describing the search process are unchanged though 8 is now interpreted as a time input. (51) and (31) imply

$$
s=\min \left(-n \gamma_{u} / \theta, 1\right)
$$

If $s$ were equal to 1 d should be raised, as it raises the consumption of the unemployed wi thout increasing their number, until either $s$ falls from unity or the constraint

$$
d<1-t=1-d \lambda / \varepsilon=1-d \lambda / g p
$$

i.e.

$$
d<g p / \lambda+g p
$$

is met. However at this point $\gamma_{u}$ would be zero. Therefore for $\theta>0$

$$
s=-\pi r_{u} / \theta
$$

at the optimum. 5

In equation (35) $\bar{c}_{i}$ is now given by

$$
\left.\begin{array}{c}
\bar{c}_{e}=r \bar{w}+(1-t)-\gamma_{e} \\
\bar{c}_{u}=r \bar{w}+d-\gamma_{u}+\theta(1-s)
\end{array}\right\}
$$

With the three changes (50) for (19), (52) for (32) and (53) in (35), the previous computations can be repeated with suitably amended parameter values.

Consider again $r=\rho=d=0$; using (50) and (52)

$$
\delta=1-\theta+\theta s=1-\theta-n \gamma_{u}
$$

while from (22) 


$$
\gamma_{\mathrm{u}}=-\dot{\varepsilon \delta /(\lambda+\varepsilon)}
$$

whe re

$$
\gamma_{u}=\frac{-\varepsilon(1-\theta)}{\lambda+\varepsilon(1-n)}
$$

and from (52)

$$
\mathbf{s}=\frac{1-\theta}{\theta} \frac{\varepsilon \eta}{\lambda+\varepsilon(1-n)}
$$

Thus if $\lambda$ is much smaller than $\varepsilon(1-n)$

$$
\begin{aligned}
\gamma_{\mathrm{u}} & \approx(1-\theta) /(1-n) \\
\mathbf{s} & \approx \frac{1-\theta}{\theta} \frac{\eta}{1-n}
\end{aligned}
$$

If $\eta=1 / 2$, its central value in section IV above, $\gamma_{u} \approx 2(1-\theta)$ while $s \approx \frac{1-\theta}{\theta}$. If $s \leq 1 / 2$ were reasonable in the absence of UI it would imply $\theta \geq 2 / 3$. We therefore consider $\theta=0.6,0.75,0.90$ $(s=2 / 3,1 / 3,1 / 9)$. Since $\varepsilon=g_{p s} s^{n}$ central values of $\eta=1 / 2$, $s=1 / 3, \varepsilon=10$ requires $g P=10 \sqrt{3} \approx 17$ so we consider $g P=10,17$, 25.

The results of these calculations are set out in table 3. The highest replacement rate is $4 \%$ in row $11(n=.8)$. The reason for the even lower replacement rates in this case seems to be that with $-Y_{u}$ less than unity in all the cases considered, the variance of wealth is much smaller than in the previous model (indeed for $\rho=r=d=0$ it falls from .72 to .05$)$; moreover with more of the utility of the unemployed coming from 'leisure' and less from dissaving interest rate effects are also weaker. However we know that, apart from savings effects on mean wealth, as $\theta \rightarrow 0 s \rightarrow 1$ so that $\mathrm{d}^{*}$ must tend towards $1-u^{*}=\frac{\varepsilon(s=1)}{\lambda+\varepsilon(s=1)}$. Since $\varepsilon(s=1)=8 \mathrm{p}$ this 1 imit is $98.837 \%$ when 


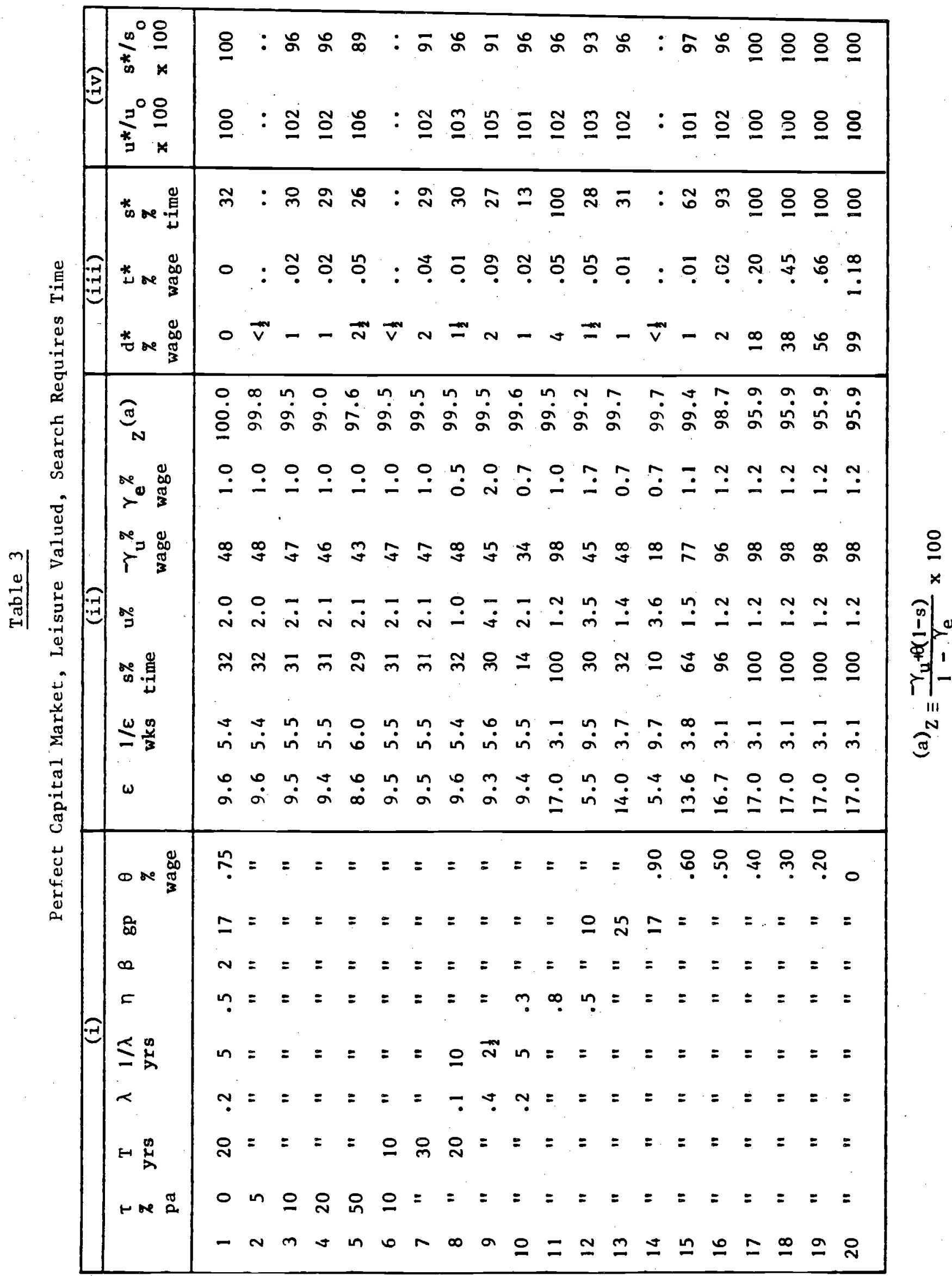


$g p=17$ and $\lambda=.02$. The course of convergence towards this limit is set out in rows $14-20$ of table 3 .

\section{VII}

As in section $V$ the absence of a capital market makes for simplification:

$$
\left.\begin{array}{c}
c_{e}=1-t \\
c_{u}=d
\end{array}\right\}
$$

If anyone works we must has $1-t>\theta$

$$
\left.\begin{array}{c}
\rho \phi_{e}=U\left(c_{e}, 0\right)+\lambda\left(\phi_{u}-\phi_{e}\right) \\
\rho \phi_{u}=U\left(C_{u}, 1-s\right)+\varepsilon\left(\phi_{e}-\phi_{u}\right) \\
u_{2}\left(C_{u},(1-s)\right)=\varepsilon^{\prime}\left(\phi_{e}-\phi_{u}\right)
\end{array}\right\}
$$

If

$$
U(C, \ell)=-\alpha e^{-\beta(C+\theta l)}
$$

then

$$
\left.\phi_{e}=-\frac{\alpha}{\rho} \frac{(\rho+\varepsilon) e^{-\beta(1-t)}+\lambda e^{-\beta(d+\theta(1-s))}}{\rho+\lambda+\varepsilon}\right)
$$

while

and

$$
\varepsilon^{\prime}=\frac{\alpha \beta \theta e^{-\beta(d+\theta(1-s))}}{\phi_{e}-\phi_{u}}=n \varepsilon / s
$$

But 


$$
\phi_{e}-\phi_{u}=-\alpha\left(e^{-\beta(1-t)}-e^{-\beta(d+\theta(1-8))}\right) /(\rho+\lambda+\varepsilon)
$$

whence

$$
\theta^{\prime}=\frac{B(\rho+\lambda+\varepsilon)}{1-e^{-B \delta}}=n \varepsilon / s
$$

which is the same as (43) except that now

$$
\delta=(1-t)-(d+\theta(1-s))=1-\theta(1-s)-d(\lambda+\varepsilon) / \varepsilon
$$

Social welfare is now

$$
S=-\alpha\left(\lambda e^{-\beta(d+\theta(1-s)}+\varepsilon e^{-\beta(1-t)} \gamma(\lambda+E)\right.
$$

Table 4 presents the results.

\section{Conclusions}

Within the family of rather special models considered here it seems that current levels of replacement $(50-80 \%$ in the U.S. and Western Europe) could only be rationalised by invoking extreme capital market imperfections. This naturally raises the question of modifying the beefit structure to introduce a loan element. As with student loans it would probably be necessary to relate repayments to subsequent earnings. As this would have further disincentive effects some combination of loans and grants is probably called for.

Although modelling intermediate cases of capital market imperfection would be difficult, variation of the benefit formula to include lump sum payments on job loss and subsidies on search would be fairly simple. Less easy, but also feasible, would be the introduction of a distribution of skills (gross wages) and the integration of UI with a 


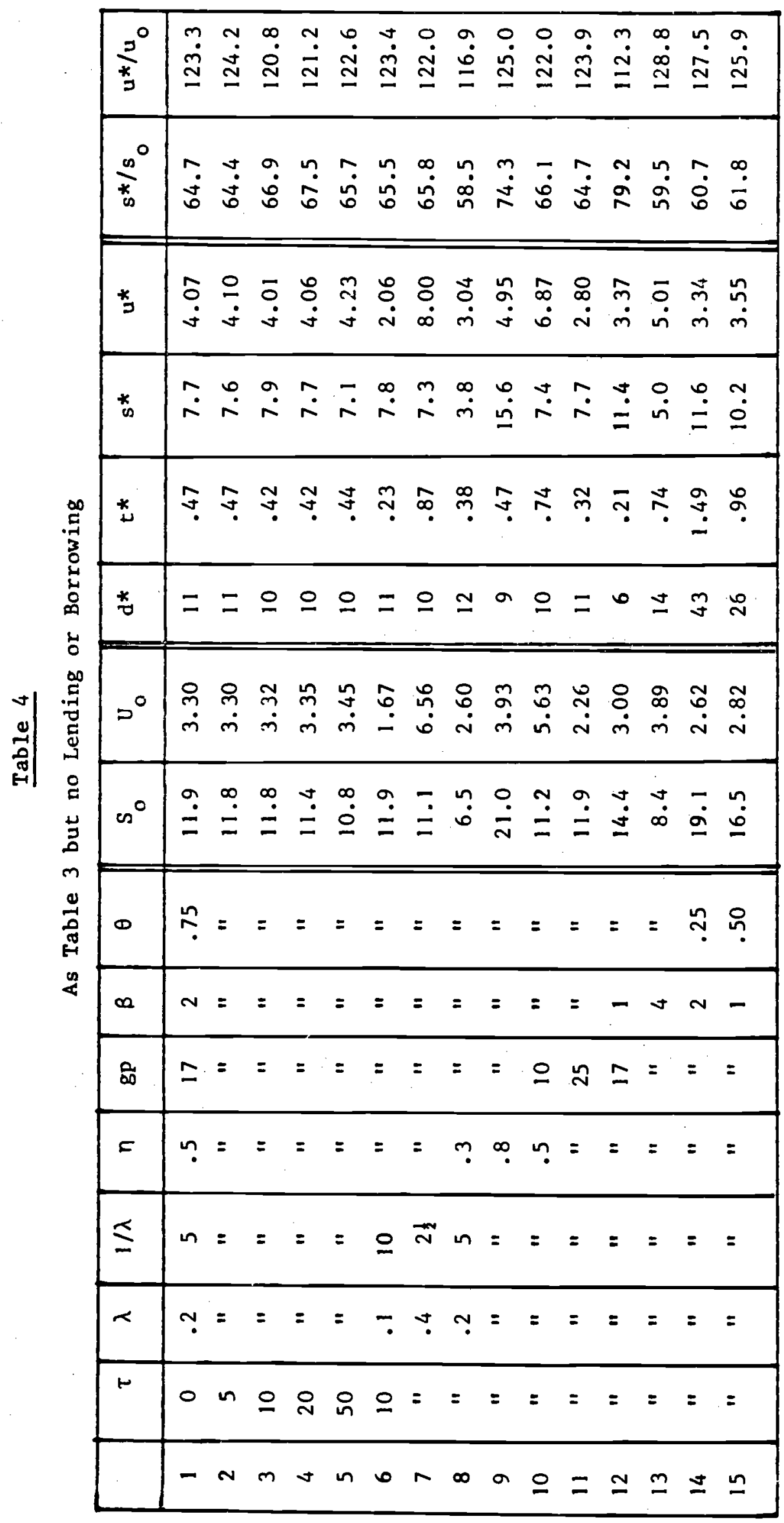


redistributive wage tax.

The models presented here relate to a population homogeneous both with respect to the wage and parameters $(\lambda)$ of job-loss and (gP and n) representing the productivity of job search. Cripps and Tarling (1974) have suggested that the distribution of the length of completed spells of unemployment implies that $\varepsilon$ is not constant in the population. In our model $\varepsilon$ would vary with the UI replacement rates but it is plausible that $g p$ and $\eta$ also depend on the characteristics of the individual and the market for his particular skills. Creedy and Disney (1977) have inferred from the distribution of the frequency of spells of unemployment that $\lambda$ is also distributed in the population.

If the population were divided into identifiable groups each characterized by its own $w, \lambda, \eta$, and $g p$, then in principle, we could identify the tax and dole appropriate to that group. However our knowledge is more likely to be represented by a joint distribution of these parameters and prescribing for that case is considerably more difficult. One possible feature of it is that we would expect the parameters applicable to the unemployed to vary with the duration of their unemployment, introducing yet another reason for relating UI benefits to duration. 


\section{Footnotes}

1. To avoid temporary lay-off unemployment the pay-roll tax is payable by fully experience-rated employers (see Feldstein (1976)).

2. We assume here that population and participation are constant. If the active population grows at the rate $v$ and all new entrants join the unemployed

$$
\dot{u}=(\lambda+\nu)(1-u)-\varepsilon u
$$

Thus in steady state where $\dot{\mathrm{u}}=0$

$$
u=\frac{\lambda+\nu}{\lambda+\nu+\varepsilon}
$$

so that $\lambda$ can be reinterpreted as the sum of the rates of job-loss and population growth.

3. This behaviour probably involves varying tha real wage rate (which we have assumed constant) as the equilibrium unemployment rate changes with $\varepsilon$ which is a function of the policy variable d.

4. Note that this implies $s<\eta / B$.

5. This argument ignores saving effects. Raising $d$ reduces aggregate savings even if search and unemployment are unaffected. For this reason the results of table 3 show several optima characterized by full time search. 


\section{References}

Atkinson, A. B., 1973, How progressive should income tax be?, in: M. Parkin, ed., Essays in modern economics (London) 90-109.

Baily, M. N., 1977, Unemployment insurance as a social insurance program (mimeo; Yale).

Creedy, J., and R. Disney, 1977, Repeated spells of unemployment in the U.K. (mimeo; Reading).

Cripps, T. F., and R. J. Tarling, 1974, An analysis of the duration of male unemployment in Great Britain 1932-73, Economic Journal 84 (June), 289-316.

Feldstein, M. S., 1976, Temporary layoffs and the theory of unemployment, Journal of Political Economy 84 (October), 937-959.

Flemning, J. S., 1977a, Optimal payroll taxes and social security funding, Journal of Public Economics 7 (August), 329-349.

Flemming, J. S., 1977b, A note on efficient unemployment insurance under progressive income taxation (mimeo; 0xford).

Hamermesh, D. S., 1977, A note on income and substitution effects in search unemployment, Economic Journal 87 (June), 312-314.

Mirrlees, J. A., 1971, An exploration of the theory of optimum income taxation, Review of Economic Studies 38 (April), 175-208.

Shavell, S., and L. Weiss, 1977, The optimal payment of unemployment insurance benefits over time, H.I.E.R. discussion paper no. 561 (June). 


\section{A NOTE ON EFFICIENT UNEMPLOYMENT INSURANCE UNDER PROGRESSIVE INCOME TAXATION}

Recent theories of unemployment such as those in Phelps et.al. (1969) emphasize the search activity of the unemployed. Expenditure of time or money in search is of the nature of investment. As with other investments one can ask whether the tax system is neutral with respect to the returns to search.

Time spent searching rather than working for current income reduces taxable income and tax paid, to this extent the Revenue subsidises search. On the other hand, if search leads to the finding of a better paid job the Revenue takes a share of the gain. If the tax is progressive the Revenue's share in the gain will exceed its share in the costs thus discouraging search. At the same time the explicit taxation of the interest returns on other investments reduces the opportunity cost of capital used up in search and thus encourages search.

In this note we formalise these statements and use them as the basis for the specification of a system of unemployment benefits designed to make the fiscal treatment of search neutral. We consider three separate cases :

(a) progressive taxation of earned income only (no interest income tax) on a continuous basis - the tax liability accrues as a progressive function of the instantaneous rate of earnings;

(b) a similar tax on the sum of earned income over a period such as a year;

(c) the continuous case (a) with income, including interest, as the tax base.

In each case we consider an unemployed person who has the opportunity to earn at the rate $W$, this represents the opportunity cost of continued search. The person believes that search for an itterval of time $\delta t$ would 
lead to an employment offer at the earnings level $W+\delta W$. Both the present opportunity and the enhanced offer are expected (correctly) to provide employment at the respective wages for a period of $T$ years after which the employee's prospects are independent of the preceding wage.

(a) On these assumptions the gain from further search is

$$
V(r, T)[N(W+\delta W)-N(W)]
$$

where $V(r, T)$ is the present value of $\mathrm{El} \mathrm{p.8.} \mathrm{For} T$ years at the tax free interest rate $r$ and $N($.$) is the net of tax wage. The cost of$ obtaining this gain is assumed to be only the net income foregone; this is the net wage $N(W)$ arising from the available offer, less the net benefit $N(B)$ for which he is eligible.

Thus the private return on searching for the extra period $\delta t$ is

$$
V(r, T) \cdot(N(W+\delta W)-N(W)) / \delta t(N(W)-N(B))
$$

while in the absence of any other distortion (i.e. wages equal marginal sccial product, $\mathrm{I}$ is the marginal social product of capital) the social return is

$$
V(r, T) \delta W / \delta t \cdot W
$$

If search is not to be distonted we need (1) and (2) to be equal, which implies

$$
\frac{N(W+\delta W)-N(W)}{\delta W}=\frac{N(W)-N(B)}{W}
$$

$N(W)$ can be written as $W(1-a(W))$ where $a(W)$ is the average tax rate. Then, if $m(W)$ is the marginal rate, the limiting value of (3) 


$$
\begin{aligned}
1-m(W) & =1-a(W)-N(B) / W \\
\frac{N(B)}{W} & =m(W)-a(W) .
\end{aligned}
$$

(4) is easily applied to a system, like that in the U.K., where there is a personal allowance ( $P$ ) of exempt income above wich a wide band of income is taxed at a constant 'basic' marginal rate b. For all levels of $W$ taxed at the basic rate

$$
\frac{N(B)}{W}=-b \cdot(W-P) / W=b P / W
$$

whence

$$
N\left(B^{*}\right)=b P \text {. }
$$

Since $b<1, \quad b P<P$ and the benefit will not be taxed. Thus the optimal gross benefit. $B^{*}=b P ; B^{*} / W$ has a maximum value of $b$ (about 1/3) at $W=P$ and declines steadily thereafter.

If the tax schedule were linear throughout, i.e. incorporated a negative income tax, and was not progressive in the marginal rate, $N(0)=b P$ and no specific unemployment benefit is called for. Thus this analysis calls for positive unemployment compensation on efficiency grounds alone under any income tax progressive in the marginal rate. However, the income tax which gives rise to this case presumably reflects a concern for redistribution which would probably raise the Baily (1977) and Flemming (1977).) optimal benefit ratio above the level required for efficiency. (See /

(b) The analysis of this case is simplified if we ignore spel1s of unemployment which stretch over two financial years. We consider a man who, having lost his job at the beginning of the year, finds himself in the position described above after a fraction $u$ of the year has passed. If $u=0$ no compensation is called for as the marginal tax rate 
in the current year, which represents the Revenue's share in the cost of search, is the same as the marginal rate in future fully employed years which is the Revenue's share in the fruits of search. If $u=1-\varepsilon$ and benefits at the rates $B(t) \quad(0<t<1)$ are taxable (3) becomes (ignoring discounting within the year)

$$
\frac{(N(W+\delta W)-N(W))}{\delta W}=\frac{N\left(\int_{0}^{1-\varepsilon} B(t) d t+\varepsilon W\right)-N\left(\int_{0}^{1} B(t) d t\right)}{\varepsilon W}
$$

or, as $\varepsilon$ and $\delta W$ tend to zero,

$$
W \cdot N^{\prime}(W)=N^{\prime}\left(\int_{0}^{1} B(t) d t\right)(W-B(1))
$$

whence

$$
\frac{B(1)}{W}=1-\frac{N^{\prime}(W)}{N^{\prime}\left(J_{B}\right)}=\frac{m(W)-m\left(\int B\right)}{1-m\left(\int B\right)}
$$

where $B(1)$ is the rate of benefit at the end of the year and $\sqrt{B}$ the cumulative benefit over the whole year. If benefits are not taxable (6) becomes

$$
\frac{B(1)}{W}=\frac{N^{\prime}(0)-N^{\prime}(W)}{N^{\prime}(0)}=\frac{m(W)-m(0)}{1-m(0)}
$$

Thus over the course of a year's unemployment the tax-free benefit rate should rise from zero to $m(W)$ if $m(0)=0$.

The result, that benefits should increase with the duration of unemployment, is contrary to the analysis of Shavell and Weiss (1977) which points out that falling benefits provide for current consumption while lending urgency to search by not underwriting future consumption. The result of the present argument would be reinforced by any tendency of $W$ to rise during unemployment as a result of search turning up opportunities superior to the previous ones but still below the 
reservation wage.

(c) If $r$ is the pre-tax interest rate $V(r, T)$ in (1) becomes $\mathrm{V}(\mathrm{r}(1-\mathrm{m}), \mathrm{T})$ while if $\mathrm{s}$ is the social rate of return $\mathrm{V}(\mathrm{r}, \mathrm{T})$ in (2) becomes $V(s, T)$ so that

$$
\frac{N(B)}{W}=1-a-(1-m) \alpha(r, s, m, T)
$$

where

$$
\alpha=\frac{s}{r(1-m)} \frac{1-e^{-r(1-m) T}}{1-e^{-s T}}
$$

(Notice that since income $(Y=W+r K)$ is now the tax base we cannot write $a$ and $m$ as depending simply on $\left.W_{.}\right)$

Since $r(i-m)$ could be either greater or less than $s$, given capital market imperfections, $\alpha$ could be either greater or less than unity.

Some light can be thrown on the implications of (8) by considering the following special cases.

(i) $r(1-m)=s, \alpha=1$. The gross interest rate $r$ exceeds $s$ by the amount of the marginal income tax.

In this case the previous result (at (a) above) is unchanged.

This case is special but not entirely absurd. The corporation tax with $100 \%$ investment relief (expensing) and interest deductibility may raise $r$ to $s /(1-c)$ where $c$ is the corporation tax rate (see Flemming (1976)), thus the case occurs when $c=m$.

(ii) $T=\infty, \alpha=s / r(1-m), N(B) / W=1-a-s / r$.

If also $s=r N(B) / W=-a$, in this case the interest tax overstimulates search in proportion to $(1-\mathrm{m})^{-1}$ while progressivity inhibits it only in proportion to (m - a) so that 
a negative replacement rate of a is required for 'neutrality'.

(iii) As $T \rightarrow 0 \quad \alpha \rightarrow 1$ and the results of section (a) go through for all $r$ and $s$.

If $T=5$ years and $r=s=10 \%$ while $m=50 \%$

$$
\alpha=1.12 \text { and } \frac{N(B)}{W}=.44-a
$$

This suggests that for realistic parameter values interest income taxation is less important than the progressivity of the tax schedule. 


\section{References}

Baily, M. N., 1977, Unemployment insurance as a social insurance program (mimeo; Yale).

Fleming, J. S., 1976 , A reappraisal of the corporation income tax, Journal of Public Economics 6, 163-169.

Flemming, J. S., 1977, Aspects of optimal unemployment insurance: search, leisure and capital market imperfections (mimeo; 0xford).

Phelps, E. S., et.al., 1969, Microeconomic foundations of unemployment and inflation theory (New York).

Shave11, S., and L. Weiss, 1977, The optimal payment of unemployment insurance benefits over time, H.I.E.R. discussion paper no. 561 (June). 